\title{
STABILITY OF LINEAR DELAY EQUATIONS UNDER A SMALL NOISE
}

\author{
by S. E. A. MOHAMMED \\ (Received 11th March 1985)
}

\section{Introduction}

The stochastic stability of linear systems driven by white noise has been treated by several authors e.g. Has'minskii [7], Kushner [11], Kleimann and Arnold [9], Pinsky, [14], Friedman and Pinsky [4], Itô and Nisio [8], Mohammed [13]. Following R. Kubo $[10,1966]$, the Brownian movement of a molecule in a "heat bath" is modelled in [13, pp. 223-226] by an asymptotically stable linear f.d.e. which is forced by white noise.

In this paper, we intend to show that global asymptotic stability to an absorbing state persists under an input noise which has a small amplitude or a short distributed memory.

\section{Preliminaries}

Consider several delays $d_{1}, d_{2}, \ldots, d_{q}, r$ such that $0 \leqq d_{i} \leqq r$ for $i=1,2, \ldots, q$. Let $J=$ $[-r, 0]$ and take as state space the Banach space $C \equiv C\left(J, \mathbb{R}^{n}\right)$ of all continuous paths $\eta: J \rightarrow \mathbb{R}^{n}$ given the supremum norm

$$
\|\eta\|_{c}=\sup \{|\eta(s)|: s \in J\}
$$

On $\mathbb{R}^{n},|\cdot|$ stands for the Euclidean norm.

Suppose $H: C \rightarrow \mathbb{R}^{n}$ is a continuous linear map such that for the deterministic linear retarded f.d.e.

$$
\left\{\begin{array}{lrl}
d y(t)=H\left(y_{t}\right) d t & t>0 \\
y_{t}(s)=y(t+s), & s \in J, & t>0 \\
y_{0}=\eta & &
\end{array}\right.
$$

the zero solution is globally asymptoticaly exponentially stable viz. the segment $y_{t} \in C$ satisfies

$$
\left\|y_{t}\right\|_{c} \leqq K e^{-\alpha t}\|\eta\|_{C}
$$

for some constants $K, \alpha>0$ and all $\eta \in C, t \geqq 0$. 
Denote by $L\left(\mathbb{R}^{m}, \mathbb{R}^{n}\right)$ the space of all linear maps $A: \mathbb{R}^{m} \rightarrow \mathbb{R}^{n}$ given the operator norm

$$
\|A\|=\sup \left\{|A(v)|: v \in \mathbb{R}^{m},|v|=1\right\} .
$$

For each complex number $\lambda \in \mathbb{C}$, define $\delta(\lambda) \in L\left(\mathbb{R}^{n}\right)$ by

$$
\delta(\lambda)(v)=\lambda v-H\left(e^{\lambda} \cdot v\right)
$$

for all $v \in \mathbb{R}^{n}$. Then $H$ is globally asymptotically exponentially stable if and only if all solutions of the characteristic equation

$$
\operatorname{det} \delta(\lambda)=0
$$

have negative real parts. (Hale [5, pp. 165-190.])

We are also given globally Lipschitz maps $g_{i}: \mathbb{R}^{n} \rightarrow L\left(\mathbb{R}^{m}, \mathbb{R}^{n}\right), 1 \leqq i \leqq q$ and $q$ independent $m$-dimensional Brownian motions $w^{i}: \mathbb{R} \geqq 0 \times \Omega \rightarrow \mathbb{R}^{m}, 1 \leqq i \leqq q$, all carried by the same filtered probability space $\left(\Omega, \mathscr{F},\left(\mathscr{F}_{t}\right)_{t \geqq 0}, P\right)$, with $w^{i}(0)=0,1 \leqq i \leqq q$.

It is known that for each $\eta \in C$ the stochastic f.d.e.

$$
\left\{\begin{array}{l}
d x(t)=H\left(x_{i}\right) d t+\sum_{i=1}^{q} g_{i}\left(x\left(t-d_{i}\right)\right) d w^{i}(t), \quad t>0 \\
x_{0}=\eta
\end{array}\right.
$$

possesses a path-wise unique sample-continuous solution ${ }^{n} x:[-r, \infty) \times \Omega \rightarrow \mathbb{R}^{n}$ adapted to the filtration $\left(\mathscr{F}_{t}\right)_{t \geqq 0}$ (Mohammed [13, Theorem II.2.1, pp. 36-40]). Observe that in (3), for each $t \geqq 0$, the segment $x_{t}: J \times \Omega \rightarrow \mathbb{R}^{n}$ is the continuous process $x_{t}(s, \omega)=$ $x(t+s, \omega), s \in J, \omega \in \Omega$. Indeed the trajectory field $\left\{{ }^{\eta} x_{t}: t \geqq 0, \eta \in C\right\}$ gives a continuous $C$-valued Feller process (Mohammed [13, Chapter III]). An absorbing state for this Feller process is a path $\eta^{0} \in C$ such that $P\left(\eta^{0} x_{t}=\eta^{0}\right)=1$ for every $t \geqq 0$. Thus $\eta^{0} \in C$ is an absorbing state for the stochastic f.d.e. (3) if and only if $\eta^{0}$ is constant and

$$
H\left(\eta^{0}\right)=0, \quad g_{i}\left(\eta^{0}(0)\right)=0
$$

for all $1 \leqq i \leqq q$ (Mohammed [13, pp. 111-112]). Indeed, every absorbing state $\eta^{0}$ must satisfy

$$
H\left(\eta^{0}\right) t+\sum_{i=1}^{q} g_{i}\left(\eta^{0}(0)\right) w^{i}(t)=0
$$

a.s. for all $t \geqq 0$. From the independence, mean and covariance properties of $w^{i}, i=1,2, \ldots, q$, we easily conclude that $H\left(\eta^{0}\right)=0, g_{i}\left(\eta^{0}(0)\right)=0$ for every $1 \leqq i \leqq q$.

Define $l>0$ by

$$
l=\max _{1 \leqq i \leqq q} \inf \left\{\frac{\left\|g_{i}\left(v_{1}\right)-g_{i}\left(v_{2}\right)\right\|}{\left|v_{1}-v_{2}\right|}: v_{1}, v_{2} \in \mathbb{R}^{n}, v_{1} \neq v_{2}\right\}
$$


Our main objective in this article is to show that, for sufficiently small values of the Lipschitz constant $l$, the stochastic f.d.e. (3) has at most one absorbing state which must be globally exponentially asymptotically stable in the mean:

\section{Theorem 1.1. Suppose}

$$
l^{2}<\frac{\alpha}{q m^{2} K^{2}}\left(\sum_{i=1}^{q} e^{2 \alpha d_{i}}\right)^{-1}
$$

Then the stochastic f.d.e. (3) has at most one absorbing state. Further, if $\eta^{0}$ is an absorbing state, then there are constants, $M, \gamma>0$, depending only on $K, \alpha, l, m, n, q, d_{i}$, and $r$ such that

$$
\left.E\right|^{n} x(t)-\left.\eta^{0}(0)\right|^{2} \leqq M\left\|\eta-\eta^{0}\right\|_{C}^{2} e^{-\gamma t}
$$

for all $t \geqq 0$ and all $\eta \in C$.

The proof of the above theorem turns upon a stochastic variation of parameters formula which we shall develop in the next section. The main idea is to construct a suitable version of a stochastic Pettis integral with values in the Banach space $C$.

\section{Stochastic variation of parameters: Stochastic Pettis integral}

Consider the stochastic f.d.e.

$$
\left\{\begin{array}{l}
d z(t)=H\left(z_{t}\right) d t+g(t) d w(t), \quad t>0 \\
z_{0}=\eta \in C
\end{array}\right.
$$

Here $H: C \rightarrow \mathbb{R}^{n}$ is any continuous linear map and $g: \mathbb{R}^{\geqq 0} \times \Omega \rightarrow L\left(\mathbb{R}^{m}, \mathbb{R}^{n}\right)$ is a measurable $\left(\mathscr{F}_{t}\right)_{t \geqq 0}$-adapted process such that, for every $a>0, \int_{0}^{a} E\|g(t, \cdot)\|^{2} d t<\infty$.

Let $T_{t}: C \rightarrow C, t \geqq 0$, denote the semi-flow of the linear r.f.d.e. $H$, viz.

$$
T_{t}(\eta)=y_{t}, \quad t \geqq 0, \quad \eta \in C
$$

By solving the r.f.d.e. (1) for bounded Borel-measurable $\eta: J \rightarrow \mathbb{R}^{n}$ it is easily seen that each $T_{t}$ extends naturally to a continuous linear operator denoted also by $T_{t}: B \rightarrow B$ on the Banach space $B \equiv B\left(J, \mathbb{R}^{n}\right)$ of all bounded Borel-measurable paths $J \rightarrow \mathbb{R}^{n}$, furnished with the supremum norm.

When $g$ is a deterministic locally square integrable function $\mathbb{R}^{\geqq 0} \rightarrow L\left(\mathbb{R}^{m}, \mathbb{R}^{n}\right)$, it is known that

$$
z_{t}=T_{t}(\eta)+\int_{0}^{t} \hat{T}_{t-u} \Delta g(u) d w(u), \quad t>0
$$

where $\Delta=\chi_{\{0\}} \operatorname{id}_{\mathbf{R}^{n}}, \quad \chi_{\{0\}}$ is the characteristic function of $\{0\}$ in $B$ and 
$\hat{T}_{t}: B\left(J, L\left(\mathbb{R}^{m}, \mathbb{R}^{n}\right)\right) \rightarrow B\left(J, L\left(\mathbb{R}^{m}, \mathbb{R}^{n}\right)\right), t \geqq 0$, is the canonical semi-flow induced by $T_{t}, t \geqq 0$. Observe that, if $\eta$ is continuous, the stochastic integral in (9) belongs almost surely to $C$. More generally a stochastic Pettis integral can be defined for $B$-valued integrand processes as follows:

Definition (Stochastic Pettis Integral)

Let $0 \leqq a \leqq b$ and $F:[a, b] \times \Omega \rightarrow B\left(J, L\left(\mathbb{R}^{m}, \mathbb{R}^{n}\right)\right)$ be a measurable map such that for

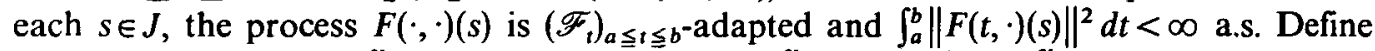
the stochastic integral $\int_{a}^{b} F(t) d w(t): J \times \Omega \rightarrow \mathbb{R}^{n}$ by $\left\{\int_{a}^{b} F(t) d w(t)\right\}(s)=\int_{a}^{b} F(t, \cdot)(s) d w(t), s \in J$.

For deterministic forced linear f.d.e.'s the variation of parameters formula is well known (Hale [5], Hale and Meyer [6]). Formula (9) is obtained in [13], through joint work with $\mathrm{H}$. v. Weizsäcker and $\mathrm{M}$. Scheutzow, by establishing a stochastic version of the deterministic formula. In what follows, we contend that the stochastic variation of parameters formula (9) still holds if $g$ is allowed to be a measurable stochastic process $\mathbb{R}^{\geqq 0} \times \Omega \rightarrow L\left(\mathbb{R}^{m}, \mathbb{R}^{n}\right)$ such that $\int_{0}^{a} E\|g(t, \cdot)\|^{2} d t<\infty$ for any $a>0$. To prove this we need several lemmas.

A two-parameter process can be approximated by a sequence of "rectangular-step" processes:

Lemma 1. Let $a \geqq 0$. Suppose $f: J \times[0, a] \times \Omega \rightarrow \mathbb{R}$ is a measurable process such that

(i) $f$ has almost all sample functions $J \times[0, a] \rightarrow \mathbb{R}$ bounded and Borel-measurable;

(ii) for each $s \in J, f(s, \cdot, \cdot):[0, a] \times \Omega \rightarrow \mathbb{R}$ is $\left(\mathscr{F}_{t}\right)_{0 \leqq t \leqq a}$-adapted;

(iii) $\int_{0}^{a} E \sup _{s \in J}|f(s, t, \cdot)|^{2} d t<\infty$.

Let $v$ be a finite Borel measure on $J$ with total variation $v(v)$. Then there is a sequence of step processes $f_{n}: J \times[0, a] \times \Omega \rightarrow \mathbb{R}, n^{\prime} \geqq 1$, each satisfying properties (i), (ii), (iii) and

$$
\lim _{n^{\prime} \rightarrow \infty} \int_{0}^{a} \int_{J} E\left|f_{n^{\prime}}(s, t, \cdot)-f(s, t, \cdot)\right|^{2} v(v)(d s) d t=0
$$

Every $f_{n}$, takes the form

$$
f_{n^{\prime}}(s, t, \omega)=\sum_{i=1}^{p} \sum_{j=1}^{l} h_{i j}(\omega) \chi_{J_{i}}(s) \chi_{I_{j}}(t), \quad s \in J, \quad t \in[0, a],
$$

$\omega \in \Omega$, where $\left\{J_{i}\right\}_{i=1}^{p},\left\{I_{j}\right\}_{j=1}^{\prime}$ are finite partitions of $J$ and $[0, a]$ consisting of disjoint intervals, and each $h_{i j} \in \mathbb{Q}^{2}\left(\Omega, \mathbb{R} ; \mathscr{F}_{t_{j}}\right), 1 \leqq i \leqq p, 1 \leqq j \leqq l$.

Proof. Let $f$ satisfy the given conditions (i), (ii) and (iii). Therefore $f$ can be viewed as a measurable, $\left(\mathscr{F}_{t}\right)_{0 \leqq t \leqq} a^{- \text {adapted, }} \mathfrak{L}^{2}(J, \mathbb{R} ; v(v))$-valued process

$$
\hat{f}:[0, a] \times \Omega \rightarrow \mathfrak{Q}^{2}(J, \mathbb{R} ; v(v))
$$

defined by

$$
f(t, \omega)(s)=f(s, t, \omega), \quad \omega \in \Omega, \quad t \in[0, a], \quad s \in J
$$


By a classical argument - suitably adapted for vector valued processes-it is not hard to

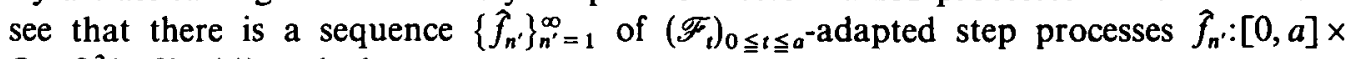
$\Omega \rightarrow \mathfrak{Q}^{2}(J, \mathfrak{R} ; v(v))$ such that

$$
\lim _{n^{\prime} \rightarrow \infty} \int_{0}^{a} E\left\|\hat{f}(t, \cdot)-\hat{f}_{n^{\prime}}(t, \cdot)\right\|_{\mathfrak{\Omega}^{2}(J)}^{2} d t=0
$$

(cf. Friedman [3, pp. 56-59]). Indeed take each

$$
\hat{f}_{n^{\prime}}(t, \omega)=\sum_{j=1}^{l} b_{j}(\omega) \chi_{I_{j}}(t), \quad \omega \in \Omega, \quad t \in[0, a]
$$

where $I_{j}=\left[t_{j}, t_{j+1}\right), 0=t_{1}<t_{2}<\cdots<t_{1+1}=a$ and $b_{j} \in \mathfrak{L}^{2}\left(\Omega, \mathfrak{L}^{2}(J, \mathbb{R} ; v(v)) ; \mathscr{F}_{t j}\right), 1 \leqq j \leqq l$. Now identify every $b_{j}$ with a (Borel $J \otimes \mathscr{F}_{t_{j}}$, Borel $\mathbb{R}$ )-measurable process $b_{j} J \cdot \times \Omega \rightarrow \mathbb{R}$ belonging to $\mathfrak{S}^{2}(J \times \Omega, \mathbb{R} ; v(v) \otimes P)$ (Dunford and Schwartz [1, pp. 196-200]). Hence there is a sequence $\left\{b_{j k}\right\}_{k=1}^{\infty}$ of Borel $J \otimes \mathscr{F}_{t_{j}}$-measurable step processes such that

$$
\lim _{k \rightarrow \infty} \int_{J} E\left|b_{j}(s, \cdot)-b_{j k}(s, \cdot)\right|^{2} v(v)(d s)=0
$$

viz.

$$
b_{j k}(s, \omega)=\sum_{i=1}^{p} a_{i j k}(\omega) \chi_{J_{i}}(s), \quad s \in J, \quad \omega \in \Omega
$$

where $a_{i j k} \in \mathfrak{L}^{2}\left(\Omega, \mathbb{R} ; \mathscr{F}_{t_{j}}\right)$, and $\left\{J_{i}\right\}_{i=1}^{p}$ is a partition of $J$ consisting of disjoint intervals.

Define the family of step processes $\left\{\hat{f}_{n^{\prime} k}: n^{\prime}, k \geqq 1\right\}$ by

$$
\hat{f}_{n^{\prime} k}(s, t, \omega)=\sum_{j=1}^{l} \sum_{i=1}^{p} a_{i j k}(\omega) \chi_{J_{i}}(s) \chi_{I_{j}}(t), \quad \omega \in \Omega, \quad s \in J, \quad t \in[0, a]
$$

Then for each fixed $n^{\prime} \geqq 1$, we have

$$
\begin{aligned}
& \int_{0}^{a} \int_{J} E\left|\hat{f}_{n^{\prime}}(t, \cdot)(s)-\hat{f}_{n^{\prime} k}(s, t, \cdot)\right|^{2} v(v)(d s) d t \\
& \quad=\int_{0}^{a} E \int_{J}\left|\sum_{j=1}^{l}\left\{b_{j}(s, \cdot)-b_{j k}(s, \cdot)\right\} \chi_{I_{j}}(t)\right|^{2} v(v)(d s) d t \\
& \quad \leqq a \sum_{j=1}^{l} \int_{J} E\left|b_{j}(s, \cdot)-b_{j k}(s, \cdot)\right|^{2} v(v)(d s) \\
& \rightarrow 0 \text { as } k \rightarrow \infty .
\end{aligned}
$$


Indeed, let $\varepsilon>0$. Then there exist $n_{0}=n_{0}(\varepsilon)>0$ and $k_{0}=k_{0}(\varepsilon)$ such that

$$
\int_{0}^{a} \int_{J} E\left|f(s, t, \cdot)-\hat{f}_{n_{0}}(t, \cdot)(s)\right|^{2} v(v)(d s) d t<\varepsilon / 2
$$

and

$$
\int_{0}^{a} \int_{J} E\left|\hat{f}_{n_{0}}(t, \cdot)(s)-\hat{f}_{n_{0} k_{0}}(s, t, \cdot)\right|^{2} v(v)(d s) d t<\varepsilon / 2
$$

Set $\hat{f}_{\varepsilon}=\hat{f}_{n_{0} k_{0}}$. Then $\hat{f}_{\varepsilon}$ is a rectangular step process of the form

$$
\hat{f}_{\varepsilon}(s, t, \omega)=\sum_{i=1}^{p} \sum_{j=1}^{l} h_{i j}(\omega) \chi_{J_{i}}(s) \chi_{I_{j}}(t), \quad \omega \in \Omega, \quad s \in J, \quad t \in[0, a],
$$

$h_{i j}=a_{i j k_{0}} \in \mathfrak{Q}^{2}\left(\Omega, \mathbb{R} ; \mathscr{F}_{t_{j}}\right)$. Moreover

$$
\int_{0}^{a} \int_{J} E\left|f(s, t, \cdot)-\hat{f}_{\varepsilon}(s, t, \cdot)\right|^{2} v(v)(d s) d t<\varepsilon
$$

Note that, for each $s \in J, \hat{f}_{\varepsilon}(s, t, \cdot)$ is $\mathscr{F}_{t}$-measurable for every $t \in[0, a]$ To obtain the required sequence of rectangular-step processes, let $\varepsilon=1 / n^{\prime}$ and $f_{n^{\prime}}=\hat{f}_{1 / n^{\prime}}$. This completes the proof of the lemma.

We now employ the above lemma to show that stochastic differentials commute with finite Borel measures viz. $d w(t) d v(s)=d v(s) d w(t)$. As a consequence of this, we deduce that our stochastic integral commutes with continuous linear maps on $B$ :

Lemma 2. Let $v$ and $f$ satisfy the conditions of Lemma 1 and $w^{\prime}$ be one-dimensional Brownian motion. Then

(i) for each $s \in J$, the Itô integral $\int_{0}^{a} f(s, t) d w^{\prime}(t)$ exists and the process $\left\{\int_{0}^{a} f(s, t) d w^{\prime}(t): s \in J\right\}$ has a measurable version with almost all sample functions $v$ integrable on $J$, i.e. the integral $\int_{J} \int_{0}^{a} f(s, t) d w^{\prime}(t) d v(s)$ exists a.s.

(ii) the repeated integral $\int_{0}^{a} \int_{J} f(s, t) d v(s) d w^{\prime}(t)$ exists and equals $\int_{J} \int_{0}^{a} f(s, t) d w^{\prime}(t) d v(s)$ a.s.

Proof. The idea is to prove the lemma for rectangular-step processes and then approximate-via Lemma 1 -in order to cover more general integrands $f$.

First take $f$ to be of the form

$$
\begin{gathered}
f(s, t, \omega)=h(\omega) \chi_{\left[\alpha_{0}, \beta_{0}\right]}(s) \chi_{\left[\gamma_{0}, \delta_{0}\right]}(t), \\
\omega \in \Omega, \quad s \in J, \quad t \in[0, a], \quad\left[a_{0}, \beta_{0}\right] \subseteq J, \quad\left[\gamma_{0}, \delta_{0}\right] \subseteq[0, a], \quad h \in \mathbb{Q}^{2}\left(\Omega, \mathbb{R} ; \mathscr{F}_{\gamma_{0}}\right)
\end{gathered}
$$


Then clearly

$$
\int_{J}^{a} \int_{0}^{a} f(s, t) d w^{\prime}(t) d v(s)=h\left[w^{\prime}\left(\delta_{0}\right)-w^{\prime}\left(\gamma_{0}\right)\right] v\left[\alpha_{0}, \beta_{0}\right]=\int_{0}^{a} \int_{J} f(s, t) d v(s) d w^{\prime}(t)
$$

By additivity of the repeated integral, the lemma holds if $f$ is any rectangular-step process.

More generally, let $f$ be any process satisfying the conditions of Lemma 1. Select a sequence of rectangular step process $f_{n^{\prime}}: J \times[0, a] \times \Omega \rightarrow \mathbb{R}, n^{\prime} \geqq 1$, as described in the above lemma. By a property of the Itô integral, the repeated integral

$$
\int_{J} \int_{\Omega}\left|\int_{0}^{a} f(s, t, \omega) d w^{\prime}(t)\right|^{2} d P(\omega) v(v)(d s)=\int_{J}^{a} \int_{0}^{a} E|f(s, t, \cdot)|^{2} d t v(v)(d s)<\infty
$$

Hence, by Fubini's Theorem (Dunford and Schwartz [1, pp. 190-200]), for a.a. $\omega \in \Omega$ the function

$$
J \ni s \mapsto\left|(\omega) \int_{0}^{a} f(s, t, \cdot) d w^{\prime}(t)\right|^{2} \in \mathbb{R}
$$

is $v(v)$-integrable. Since $v(v)$ is finite, then the function

$$
J \ni s \mapsto(\omega) \int_{0}^{a} f(s, t, \cdot) d w^{\prime}(t) \in \mathbb{R}
$$

is $v$-integrable for a.a. $\omega \in \Omega$; viz. the process $\left\{\int_{0}^{a} f(s, t) d w^{\prime}(t): s \in J\right\}$ has a (unique up-toequivalence) measurable version $J \times \Omega \rightarrow \mathbb{R}$ with almost all sample functions $v$-integrable on $J$, i.e., $\int_{J} \int_{0}^{a} f(s, t) d w^{\prime}(t) d v(s)<\infty$ a.s. (Dunford and Schwartz [1, pp. 196-200]).

Because $f$ has bounded Borel-measurable sample functions, the integral $\int_{J} f(s, t) d v(s)$ exists a.s. for every $t \in[0, a]$. Furthermore, for each $t \in[0, a]$,

$$
E\left|\int_{J} f(s, t) d v(s)\right|^{2} \leqq v(v)(J) \int_{J} E|f(s, t)|^{2} v(v)(d s)<\infty
$$

by Hölder's inequality and Fubini's Theorem. So

$$
\int_{0}^{a} E\left|\int_{J} f(s, t) d v(s)\right|^{2} d t \leqq v(v)(J) \int_{0}^{a} \int_{J} E|f(s, t)|^{2} v(v)(d s) d t<\infty
$$

Therefore the stochastic integral $\int_{0}^{a} \int_{J} f(s, t) d v(s) d w^{\prime}(t)$ is well-defined. 
Now look at the expression

$$
\begin{aligned}
E\left|\int_{0}^{a} \int_{J} f(s, t) d v(s) d w^{\prime}(t)-\int_{0}^{a} \int_{J} f_{n^{\prime}}(s, t) d v(s) d w^{\prime}(t)\right|^{2} \\
=\int_{0}^{a} E\left|\int_{J}\left\{f(s, t)-f_{n^{\prime}}(s, t)\right\} d v(s)\right|^{2} d t \\
\quad \leqq \int_{0}^{a} \int_{J} E\left|f(s, t)-f_{n^{\prime}}(s, t)\right|^{2} v(v)(d s) d t \rightarrow 0 \text { as } n^{\prime} \rightarrow \infty
\end{aligned}
$$

i.e.

$$
\int_{0}^{a} \int_{J} f(s, t) d v(s) d w^{\prime}(t)=\lim _{n^{\prime} \rightarrow \infty} \int_{0}^{a} \int_{J} f_{n^{\prime}}(s, t) d v(s) d w^{\prime}(t)
$$

in $\mathfrak{L}^{\mathbf{2}}$.

By a similar argument, it is easily seen that

$$
\int_{J} \int_{0}^{a} f(s, t) d w^{\prime}(t) d v(s)=\lim _{n^{\prime} \rightarrow \infty} \int_{J}^{a} \int_{0}^{a} f_{n^{\prime}}(s, t) d w^{\prime}(t) d v(s)
$$

Complete the proof by noting that a.s.

$$
\int_{0}^{a} \int_{J} f_{n^{\prime}}(s, t) d v(s) d w^{\prime}(t)=\iint_{J}^{a} \int_{0}^{a} f_{n^{\prime}}(s, t) d w^{\prime}(t) d v(s)
$$

for every $n^{\prime} \geqq 1$.

Remark. By reducing to coordinates, the above lemma generalizes immediately to $L\left(\mathbb{R}^{m}, \mathbb{R}^{n}\right)$-valued $f, L\left(\mathbb{R}^{n}\right)$-valued $v$ and $n$-dimensional $w^{\prime}$.

Lemma 3. Denote by the same symbol $H: B \rightarrow \mathbb{R}^{n}$ the natural (unique) continuous linear extension of $H: C \rightarrow \mathbb{R}^{n}$, obtained via the Riesz representation theorem.

Suppose $F:[0, a] \times \Omega \rightarrow B\left(J, L\left(\mathbb{R}^{m}, \mathbb{R}^{n}\right)\right)$ is measurable, $\left(\mathscr{F}_{t}\right)_{0 \leqq t \leq a}$-adapted and satisfies the conditions

(i) $\sup _{a}\{\|F(t, \cdot)(s)\|: t \in[0, a], s \in J\}<\infty$ a.s.

(ii) $\int_{0}^{a} E\|F(t, \cdot)\|_{B}^{2} d t<\infty$.

Let $\hat{H}: B\left(J, L\left(\mathbb{R}^{m}, \mathbb{R}^{n}\right)\right) \rightarrow L\left(^{m}, \mathbb{R}^{n}\right)$ be the continuous linear map induced by $H$.

If $\int_{0}^{a} F(t) d w(t)$ has a.a. sample paths in $B$, then

$$
H\left[\int_{0}^{a} F(t) d w(t)\right]=\int_{0}^{a} \hat{H}[F(t)] d w(t) \text { a.s. }
$$


Proof. By the Riesz representation theorem, $H$ corresponds to a Borel, $L\left(\mathbb{R}^{n}\right)$-valued measure $\mu$; viz.

$$
H(\eta)=\int_{J} d \mu(s)(\eta(s)), \quad \eta \in B
$$

The map $\hat{H}: B\left(J, L\left(\mathbb{R}^{m}, \mathbb{R}^{n}\right)\right) \rightarrow L\left(\mathbb{R}^{m}, \mathbb{R}^{n}\right)$ is induced by $H$ through the formula

$$
\hat{H}(\xi)(v)=H[\xi(\cdot)(v)], \quad \xi \in B\left(J, L\left(\mathbb{R}^{m}, \mathbb{R}^{n}\right)\right), \quad v \in \mathbb{R}^{m} .
$$

Equivalently,

$$
\hat{H}(\xi)=\int_{J} d \mu(s) \circ \xi(s), \xi \in B\left(J, L\left(\mathbb{R}^{m}, \mathbb{R}^{n}\right)\right) .
$$

It is clear that $\hat{H}$ is continuous linear, with $\|\hat{H}\| \leqq\|H\|$. Since $F$ has almost all sample paths bounded, then so does the process $[0, a] \ni t \mapsto \hat{H}[F(t)] \in L\left(\mathbb{R}^{m}, \mathbb{R}^{n}\right)$. Thus the integral $\int_{0}^{a} \hat{H}[F(t)] d w(t)$ is well-defined. So by Lemma 2 we get

$$
\begin{aligned}
H\left[\int_{0}^{a} F(t) d w(t)\right] & =\int_{J} d \mu(s)\left(\left\{\int_{0}^{a} F(t) d w(t)\right\}(s)\right)=\int_{J} d \mu(s)\left(\int_{0}^{a} F(t)(s) d w(t)\right) \\
& =\int_{0}^{a} \int_{J} d \mu(s) \circ F(t)(s) d w(t)=\int_{0}^{a} \hat{H}[F(t)] d w(t) \quad \text { a.s. }
\end{aligned}
$$
form

Remark. It is not hard to see that each $T_{t}$ and $\widehat{T}_{t}, t>0$, admit representations of the

$$
\begin{aligned}
& T_{t}(\eta)=\int_{J} d v_{t}(s)(\eta(s)), \quad \eta \in B \\
& \hat{T}_{t}(\xi)=\int_{J} d v_{t}(s) \circ \xi(s), \quad \xi \in B\left(J, L\left(\mathbb{R}^{m}, \mathbb{R}^{n}\right)\right)
\end{aligned}
$$

where $v_{t}$ is a $B\left(J, L\left(\mathbb{R}^{n}\right)\right)$-valued measure. Under the conditions of the above lemma, it therefore follows that, for each $t \geqq 0, \int_{0}^{a} \hat{T}_{t}[F(u)] d w(u)$ is a version of $T_{t}\left[\int_{0}^{a} F(u) d w(u)\right]$.

The following lemma is probably well-known. The same symbol $\widehat{T}_{t}, t \geqq 0$, is used to denote the induced semi-flow(s) by $T_{t}, t \geqq 0$, on $B\left(J, L\left(\mathbb{R}^{m}, \mathbb{R}^{n}\right)\right)$ or $B\left(J, L\left(\mathbb{R}^{n}\right)\right)$.

Lemma 4. The semi-flow $\left\{\hat{T}_{t}\right\}_{t \geqq 0}$ satisfies the identities

$$
\begin{gathered}
\frac{d}{d t} \widehat{T}_{t}(\xi)(0)=\hat{H}\left[\widehat{T}_{t}(\xi)\right], t>0, \xi \in B\left(J, L\left(\mathbb{R}^{m}, \mathbb{R}^{n}\right)\right) \\
\hat{T}_{t_{1}+t_{2}}=\widehat{T}_{t_{1}} \circ \hat{T}_{t_{2}}, \quad t_{1}, t_{2} \geqq 0, \quad \hat{T}_{0}=\mathrm{id} \\
\hat{T}_{t}(\xi A)=\hat{T}_{t}(\xi) \circ A, \quad A \in L\left(\mathbb{R}^{m}, \mathbb{R}^{n}\right), \quad \xi \in B\left(J, L\left(\mathbb{R}^{n}\right)\right) .
\end{gathered}
$$


Proof. Recall that $\hat{H}$ and $\hat{T}_{t}, t \geqq 0$, are defined by

$$
\begin{gathered}
\hat{H}(\xi)(v)=H[\xi(\cdot)(v)], \\
\hat{T}_{t}(\xi)(s)(v)=\left[T_{t}\{\xi(\cdot)(v)\}\right](s)
\end{gathered}
$$

for $\xi \in B\left(J, L\left(\mathbb{R}^{m}, \mathbb{R}^{n}\right)\right), v \in \mathbb{R}^{m}, s \in J$. It is easy to see that

$$
\widehat{T}_{t}(\xi)(s)= \begin{cases}\hat{T}_{t+s}(\xi)(0), & t+s \geqq 0 \\ \xi(t+s), & -r \leqq t+s<0\end{cases}
$$

Therefore the path

$$
[-r, \infty) \ni t \mapsto \begin{cases}\hat{T}_{r}(\xi)(0), & t>0 \\ \xi(t), & t \in J\end{cases}
$$

corresponds to the unique solution of $\hat{H}$ through $\xi$, because

$$
\frac{d}{d t} \widehat{T}_{t}(\xi)(0)(v)=\frac{d}{d t}\left[T_{t}\{\xi(\cdot)(v)\}\right](0)=H\left[T_{t}\{\xi(\cdot)(v)\}\right]=\hat{H}\left[\hat{T}_{t}(\xi)\right](v)
$$

This proves identity (10).

The semi-group property (11) follows directly from the definition of $\hat{T}_{t}$, or alternatively from the continuation property of the r.f.d.e. (10).

It remains to demonstrate (12). Let $A \in L\left(\mathbb{R}^{m}, \mathbb{R}^{n}\right), \xi \in B\left(J, L\left(\mathbb{R}^{n}\right)\right)$. Then the path

$$
\begin{gathered}
\xi A: J \rightarrow L\left(\mathbb{R}^{m}, \mathbb{R}^{n}\right) \\
s \mapsto \xi(s) \circ A
\end{gathered}
$$

belongs to $B\left(J, L\left(\mathbb{R}^{m}, \mathbb{R}^{n}\right)\right)$. Then, for $s \in J, v \in \mathbb{R}^{m}$,

$$
\hat{T}_{i}(\xi A)(s)(v)=\left[T_{t}\{\xi(\cdot)(A(v))\}\right](s)=\left[\hat{T}_{t}(\xi)(s) \circ A\right](v)
$$

Hence (12) holds, and the proof of the lemma is complete.

Our final lemma in this section tells us how to "differentiate inside a stochastic integral sign":

Lemma 5. Let $f:[0, a] \times[0, a] \rightarrow L\left(\mathbb{R}^{m}, \mathbb{R}^{n}\right)$ and its partial derivative $(t, u) \mapsto(\partial / \partial t) f(t, u)$ be continuous on the triangle $T=\{(t, u): 0 \leqq u \leqq t \leqq a\}$. Suppose $\tilde{z}$ is a sample continuous process such that the stochastic (McShane) integral

$$
X(t)=\int_{0}^{t} f(t, u) d \tilde{z}(u), \quad t \in[0, a]
$$


exists. Then $X$ is a sample continuous semi-martingale and

$$
d X(t)=f(t, t) d \tilde{z}(t)+\left\{\int_{0}^{t} \frac{\partial}{\partial t} f(t, u) d \tilde{z}(u)\right\} d t, \quad 0<t<a .
$$

Proof. Without loss of generality, assume that $\tilde{z}(0)=0$ a.s.

Suppose first that $f$ is $C^{2}$ on the triangle $T$. Define the process $X:[0, a] \times \Omega \rightarrow \mathbb{R}^{n}$ by

$$
X(t)=\int_{0}^{t} f(t, u) d \tilde{z}(u), \quad 0 \leqq t \leqq a
$$

We may integrate by parts (Elworthy $[2, \mathrm{pp} .79-80])$ to get

$$
X(t)=\tilde{z}(t) f(t, t)-\int_{0}^{t} \tilde{z}(u) \frac{\partial}{\partial u} f(t, u) d u, \quad 0 \leqq t \leqq a .
$$

Taking stochastic differentials gives

$$
d X(t)=f(t, t) d \tilde{z}(t)+\left\{\tilde{z}(t) \frac{d}{d t} f(t, t)\right\} d t-\left.\tilde{z}(t) \frac{\partial}{\partial u} f(t, u)\right|_{u=t} d t-\left\{\int_{0}^{t} \tilde{z}(u) \frac{\partial}{\partial t} \frac{\partial}{\partial u} f(t, u) d u\right\} d t
$$

Using the fact that $f$ is $C^{2}$ and the Chain Rule, one gets

$$
\begin{aligned}
d X(t) & =f(t, t) d \tilde{z}(t)+\left.\tilde{z}(t) \frac{\partial}{\partial t} f(t, u)\right|_{u=t} d t-\left\{\int_{0}^{t} \tilde{z}(u) \frac{\partial}{\partial u} \frac{\partial}{\partial t} f(t, u) d u\right\} d t \\
& =f(t, t) d \tilde{z}(t)+\left\{\int_{0}^{t} \frac{\partial}{\partial t} f(t, u) d \tilde{z}(u)\right\} d t .
\end{aligned}
$$

To prove the lemma for continuous $f$ with continuous first partial derivative on $T$, select a sequence of $C^{2}$ (or even $C^{\infty}$ ) functions $f_{k}: T \rightarrow L\left(\mathbb{R}^{m}, \mathbb{R}^{n}\right), k \geqq 1$, such that

$$
f_{k}(t, u) \rightarrow f(t, u)
$$

and

$$
\frac{\partial}{\partial t} f_{k}(t, u) \rightarrow \frac{\partial}{\partial t} f(t, u)
$$

as $k \rightarrow \infty$ uniformly for $(t, u) \in T$. This can be done as follows: pick two sequences of smooth functions $\left\{h_{k}^{1}\right\}_{k=1}^{\infty},\left\{h_{k}^{2}\right\}_{k=1}^{\infty}$ such that $h_{k}^{1}(t, u) \rightarrow(\partial / \partial t) f(t, u)$ and $h_{k}^{2}(u, u) \rightarrow f(u, u)$ as $k \rightarrow \infty$, uniformly for $(t, u),(u, u)$. Define the required sequence $\left\{f_{k}\right\}_{k=1}^{\infty}$ by

$$
f_{k}(t, u)=h_{k}^{2}(u, u)+\int_{u}^{t} h_{k}^{1}(v, u) d v, \quad(t, u) \in T, \quad k \geqq 1 .
$$


Now let $\left\{X^{k}\right\}_{k=1}^{\infty}$ be the sequence of processes

$$
X^{k}(t)=\int_{0}^{t} f_{k}(t, u) d \tilde{z}(u), \quad k \geqq 1, t \in[0, a]
$$

Then, by what has already been proved,

$$
X^{k}(t)=\int_{0}^{t} f_{k}(u, u) d \tilde{z}(u)+\int_{0}^{t}\left\{\int_{0}^{v} \frac{\partial}{\partial v} f_{k}(v, u) d \tilde{z}(u)\right\} d v
$$

for all $t \in[0, a]$ and $k \geqq 1$. But, for each $t \in[0, a]$,

$$
E\left|\int_{0}^{t} f_{k}(u, u) d \tilde{z}(u)-\int_{0}^{t} f(u, u) d \tilde{z}(u)\right|^{2} \leqq M \int_{0}^{t}\left|f_{k}(u, u)-f(u, u)\right|^{2} d u \rightarrow 0 \quad \text { as } k \rightarrow \infty
$$

and

$$
\begin{gathered}
E\left|\int_{0}^{t} \int_{0}^{v} \frac{\partial}{\partial v} f_{k}(v, u) d \tilde{z}(u) d v-\int_{0}^{t} \int_{0}^{v} \frac{\partial}{\partial v} f(v, u) d \tilde{z}(u) d v\right|^{2} \\
\quad \leqq t \int_{0}^{t} E\left|\int_{0}^{v}\left\{\frac{\partial}{\partial v} f_{k}(v, u)-\frac{\partial}{\partial v} f(v, u)\right\} d \tilde{z}(u)\right|^{2} d v \\
\leqq M t \int_{0}^{t} \int_{0}^{v}\left|\frac{\partial}{\partial v} f_{k}(v, u)-\frac{\partial}{\partial v} f(v, u)\right|^{2} d u d v \\
\rightarrow 0 \text { as } \quad k \rightarrow \infty,
\end{gathered}
$$

by uniform convergence, where $M>0$ is some constant. Similarly,

$$
E\left|X(t)-X^{k}(t)\right|^{2}=E\left|\int_{0}^{t}\left\{f(t, u)-f_{k}(t, u)\right\} d \tilde{z}(u)\right|^{2} \rightarrow 0 \text { as } k \rightarrow \infty \text {, for each } t \in[0, a] \text {. }
$$

Therefore, for each $t \in[0, a]$, we can take $\mathfrak{L}^{2}$-limits as $k \rightarrow \infty$ in (15) to get

$$
X(t)=\int_{0}^{t} f(u, u) d \tilde{z}(u)+\int_{0}^{t} \int_{0}^{v} \frac{\partial}{\partial v} f(v, u) d \tilde{z}(u) d v
$$

The lemma is proved.

The way is now clear to prove the stochastic variation of parameters formula for our stochastic f.d.e. (7):

Theorem 3.1. With the given conditions on $H$ and $g$, the trajectory of the stochastic 
f.d.e. (7) satisfies

$$
z_{t}=T_{t}(\eta)+\int_{0}^{t} \widehat{T}_{t-u} \Delta g(u) d w(u), \quad t \geqq 0
$$

where $\Delta=\chi_{10\}} \mathrm{id}_{\mathbf{R}^{n}}$.

Proof. Fix any $a>0$. Assume first that for each $t \in[0, a], g(t, \cdot) \in \mathfrak{L}^{2}\left(\Omega, L\left(\mathbb{R}^{m}, \mathbb{R}^{n}\right)\right)$ and the function

$$
[0, a] \ni t \mapsto E\|g(t, \cdot)\|^{2} \in \mathbb{R}
$$

is (Lebesgue) a.e. continuous and bounded.

Define the process $Y:[-r, a] \times \Omega \rightarrow \mathbb{R}^{n}$ by

$$
Y(t)= \begin{cases}T_{t}(\eta)(0)+\int_{0}^{t}\left\{\hat{T}_{t-u} \Delta g(u)\right\}(0) d w(u), & 0 \leqq t \leqq a \\ \eta(t), & t \in J\end{cases}
$$

Then

$$
Y(t)=T_{t}(\eta)(0)+\int_{0}^{t}\left(\hat{T}_{t-u} \Delta\right)(0) g(u) d w(u), \quad 0 \leqq t \leqq a .
$$

Set $\tilde{z}(t)=\int_{0}^{t} g(u) d w(u), t \geqq 0$. Then $\tilde{z}$ is a martingale and the map $[0, a] \ni t \mapsto \tilde{z}(t) \in \mathfrak{Q}^{2}\left(\Omega, \mathbb{R}^{n}\right)$ is $\frac{1}{2}$-Hölder continuous. Therefore the substitution formula (McShane [12, pp. 134-138]) gives

$$
Y(t)=T_{t}(\eta)(0)+\int_{0}^{t}\left(\widehat{T}_{t-u} \Delta\right)(0) d \tilde{z}(u), \quad 0 \leqq t \leqq a
$$

Using Lemma 5 we can take differentials in (18) to get

$$
\begin{aligned}
d Y(t) & =H\left(T_{t}(\eta)\right) d t+d \tilde{z}(t)+\int_{0}^{t} \frac{\partial}{\partial t}\left(\hat{T}_{t-u} \Delta\right)(0) d \tilde{z}(u) d t \\
& =H\left(T_{t}(\eta)\right) d t+g(t) d w(t)+\left\{\int_{0}^{t} \hat{H}\left(\hat{T}_{t-u} \Delta\right) g(u) d w(u)\right\} d t \\
& =H\left(T_{t}(\eta)\right) d t+g(t) d w(t)+\left\{\int_{0}^{t} \hat{H}\left[\hat{T}_{t-u} \Delta g(u)\right] d w(u)\right\} d t \\
& =H\left(T_{t}(\eta)\right) d t+g(t) d w(t)+H\left[\int_{0}^{t} \hat{T}_{t-u} \Delta g(u) d w(u)\right] d t \\
& =H\left[T_{t}(\eta)+\int_{0}^{t} \hat{T}_{t-u} \Delta g(u) d w(u)\right] d t+g(t) d w(t), \quad t>0
\end{aligned}
$$

where we have used Lemmas 3 and 4. 
Now observe that

$$
Y_{t}=T_{t}(\eta)+\int_{0}^{t} \hat{T}_{t-u} \Delta g(u) d w(u), \quad t \geqq 0
$$

so (19) becomes

$$
\begin{aligned}
d Y(t) & =H\left(Y_{t}\right) d t+g(t) d w(t), \quad t>0 \\
Y_{0} & =\eta
\end{aligned}
$$

i.e., $Y$ is the unique $\left(\mathscr{F}_{t}\right)_{t \geqq 0}$-adapted continuous solution of the stochastic f.d.e. (7) through $\eta \in C$; so a.s.

$$
z_{t}=Y_{t}=T_{t}(\eta)+\int_{0}^{t} \widehat{T}_{t-u} \Delta g(u) d w(u)
$$

(Mohammed [13, pp. 36-39]).

Finally, let $g$ be measurable, $\left(\mathscr{F}_{t}\right)_{0 \leqq t \leqq a}$-adapted and such that $\int_{0}^{a} E\|g(t, \cdot)\|^{2} d t<\infty$ for every $a>0$.

Pick a sequence of measurable, $\left(\mathscr{F}_{t}\right)_{0 \leqq t \leqq}$-adapted processes $g_{k}:[0, a] \times \Omega \rightarrow L\left(\mathbb{R}^{m}, \mathbb{R}^{n}\right)$, $k \geqq 1$, such that

(i) for each $k \geqq 1, \int_{0}^{a} E\left\|g_{k}(t, \cdot)\right\|^{2} d t<\infty$;

(ii) for each $k \geqq 1$, the function

$$
[0, a] \ni t \mapsto E\left\|g_{k}(t, \cdot)\right\|^{2} \in \mathbb{R}
$$

is continuous (Lebesgue) a.e. and is bounded;

(iii) $\lim _{k \rightarrow \infty} \int_{0}^{a} E\left\|g_{k}(t, \cdot)-g(t, \cdot)\right\|^{2} d t=0$.

For each $k \geqq 1$, let $z^{k}:[-r, a] \times \Omega \rightarrow \mathbb{R}^{n}$ be the unique solution of

$$
\left\{\begin{aligned}
d z^{k}(t) & =H\left(z_{t}^{k}\right)+g_{k}(t) d w(t), \quad 0<t<a \\
z_{0}^{k} & =\eta
\end{aligned}\right.
$$

Employing (7) and (21), a simple applicatıon of Gronwall's lemma gives $K_{1}>0$ such that

$$
E\left\|z_{t}^{k}-z_{t}\right\|_{C}^{2} \leqq K_{1}\left\{\int_{0}^{a} E\left\|g_{k}(u)-g(u)\right\|^{2} d u\right\} e^{2 a\|H\|^{2} t} \rightarrow 0, \text { as } k \rightarrow \infty \text { for each } t \in[0, a] .
$$

Now, by the first part of this proof, each $z^{k}$ satisfies

$$
z_{t}^{k}=T_{t}(\eta)+\int_{0}^{t} \widehat{T}_{t-u} \Delta g_{k}(u) d w(u), \quad 0 \leqq t \leqq a
$$

for $k \geqq 1$. 
If we evaluate (22) at any $s \in J$ and let $k \rightarrow \infty$ in $\mathfrak{L}^{2}$, we immediately get from property (iii) of the $g_{k}$ that

$$
\begin{aligned}
z_{t}(s) & =\lim _{k \rightarrow \infty} z_{t}^{k}(s)=T_{t}(\eta)(s)+\lim _{k \rightarrow \infty} \int_{0}^{t}\left(\hat{T}_{t-u} \Delta\right)(s) g_{k}(u) d w(u) \\
& =T_{t}(\eta)(s)+\int_{0}^{t}\left(\hat{T}_{t-u} \Delta\right)(s) g(u) d w(u)
\end{aligned}
$$

Since $z$ has continuous sample paths (Mohammed [13, pp. 36-39]), the equivalence (23) implies that the integral $\int_{0}^{t} \widehat{T}_{t-u} \Delta g(u) d w(u)$ has almost all sample paths continuous and the formula (9) then holds on a set of full $P$-measure which is independent of $t \in \mathbb{R} \geq 0$ and $s \in J$. This concludes the proof of the theorem.

\section{Asymptotic stability of absorbing states}

In this section we employ the stochastic variation of parameters formula to give a proof of Theorem 1.1.

Recall the notations and assumptions of Section 2. The following lemma on the asymptotic stability of $\hat{H}$ is well-known (Hale [5, pp. 185-187]):

Lemma. Suppose $H$ is asymptotically stable with constants $K, \alpha>0$ satisfying inequality (2) viz.

$$
\left\|T_{i}(\eta)\right\|_{C} \leqq K\|\eta\|_{c} e^{-\alpha t}, \quad t \geqq 0, \quad \eta \in C
$$

Then

$$
\left\|\widehat{T}_{t}\left(\chi_{\{0\}} A\right)\right\|_{B} \leqq K\|A\| e^{-\alpha t}
$$

for all $t \geqq 0$ and all $A \in L\left(\mathbb{R}^{m}, \mathbb{R}^{n}\right)$.

Proof. Approximate $\chi_{10\}}: J \rightarrow \mathbb{R}$ pointwise by a sequence of continuous functions $\left\{\eta^{k}\right\}_{k=1}^{\infty}$ such that

$$
\chi_{\{0\}}(s)=\lim _{k \rightarrow \infty} \eta^{k}(s), \quad s \in J
$$

and $\left\|\eta^{k}\right\|_{c} \leqq 1$ for all $k \geqq 1$, e.g.

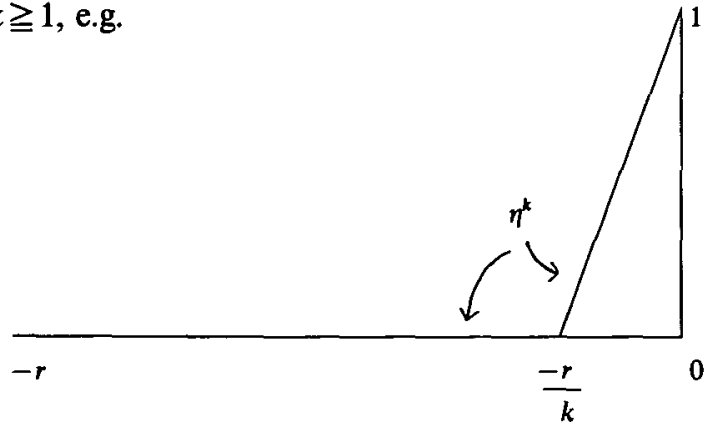


Let $A \in L\left(\mathbb{R}^{m}, \mathbb{R}^{n}\right), v \in \mathbb{R}^{m}, \quad s \in J$. Using (2) and the weak continuity of $T_{t}, t \geqq 0$ (Mohammed [13, pp. 193-194]), we get

$$
\hat{T}_{t}\left(\chi_{\{0\}} A\right)(s)(v)=\lim _{k \rightarrow \infty}\left[T_{t}\left\{\eta^{k}(\cdot) A(v)\right\}\right](s) \leqq K|A(v)| e^{-\alpha t}
$$

Therefore

$$
\left\|\widehat{T}_{t}\left(\chi_{\{0\}} A\right)\right\|_{B}=\sup _{s \in J} \sup _{\substack{v \in R^{n} \\|v|=1}}\left|\widehat{T}_{t}\left(\chi_{\{0\}} A\right)(s)(v)\right| \leqslant K\|A\| e^{-\alpha t}
$$

for all $t \geqq 0$. This proves the lemma.

Proof of Theorem 1.1. Suppose $H$ is asymptotically stable and let

$$
l^{2}<\frac{\alpha}{q m^{2} K^{2}}\left(\sum_{i=1}^{q} e^{2 \alpha d_{i}}\right)^{-1}
$$

For any $\eta \in C,{ }^{n} x:[-r, \infty) \times \Omega \rightarrow \mathbb{R}^{n}$ is the unique solution of

$$
\left\{\begin{aligned}
d^{\eta} x(t) & =H\left({ }^{\eta} x_{t}\right) d t+\sum_{i=1}^{q} g_{i}\left({ }^{\eta} x\left(t-d_{i}\right)\right) d w^{i}(t), \quad t>0 \\
x_{0} & =\eta
\end{aligned}\right.
$$

Since the Brownian motions $\left\{w^{i}\right\}_{i=1}^{q}$ are independent, then the process $w=\bigoplus_{i=1}^{q} w^{i}$ : $\mathbb{R}^{\geqq 0} \times \Omega \rightarrow\left(\mathbb{R}^{m}\right)^{q}$ is an $m q$-dimensional Brownian motion on $\left(\Omega, \mathscr{F},\left(\mathscr{F}_{t}\right)_{t \geqq 0}, P\right)$. Define the processes $\tilde{g}_{i}: \mathbb{R}^{\geqq 0} \times \Omega \rightarrow L\left(\mathbb{R}^{m}, \mathbb{R}^{n}\right), \tilde{g}: \mathbb{R}^{\geqq 0} \times \Omega \rightarrow L\left(\left(\mathbb{R}^{m}\right)^{q}, \mathbb{R}^{n}\right)$ by

$$
\begin{aligned}
\tilde{g}_{i}(t) & =g_{i}\left({ }^{\eta} x\left(t-d_{i}\right)\right), \quad i=1,2, \ldots, q \\
\tilde{g} & =\bigoplus_{i=1}^{q} \tilde{g}_{i} .
\end{aligned}
$$

Then (3) takes the form

$$
d^{n} x(t)=H\left({ }^{n} x_{t}\right)+\tilde{g}(t) d w(t)
$$

Note that the Lipschitz property of the $g_{i}$ 's implies that $\tilde{g}$ is a measurable $\left(\mathscr{F}_{t}\right)_{t \geqq 0^{-}}$ adapted process satisfying the conditions of Theorem 3.1. Thus we obtain

$$
{ }^{\eta} x_{t}=T_{t}(\eta)+\sum_{i=1}^{q} \int_{0}^{1} \hat{T}_{t-u} \Delta g_{i}\left({ }^{\eta} x\left(u-d_{i}\right)\right) d w^{i}(u), \quad t \geqq 0
$$


If $\eta^{\circ}$ is any absorbing state for (3), it therefore follows that, for $t \geqq r$, we have

$$
\begin{aligned}
\left.E\right|^{\eta} x(t) & -\left.\eta^{0}(0)\right|^{2} \leqq 2\left|\left\{T_{t}\left(\eta-\eta^{0}\right)\right\}(0)\right|^{2} \\
& +2 q \sum_{i=1}^{q} E\left|\int_{0}^{t}\left(\hat{T}_{t-u} \Delta\right)(0)\left\{g_{i}\left({ }^{\eta} x\left(u-d_{i}\right)\right)-g_{i}\left(\eta^{0}(0)\right)\right\} d w^{i}(u)\right|^{2} \\
\leqq & 2 K^{2} e^{-2 \alpha t}\left\|\eta-\eta^{0}\right\|_{C}^{2}+\left.2 q m^{2} l^{2} K^{2} e^{-2 \alpha t} \sum_{i=1}^{q} \int_{0}^{t} e^{2 \alpha u} E\right|^{\eta} x\left(u-d_{i}\right)-\left.\eta^{0}(0)\right|^{2} d u \\
= & \left.2 K^{2} e^{-2 \alpha t}\left\|\eta-\eta^{0}\right\|\right|_{c} ^{2}+2 q m^{2} l^{2} K^{2} e^{-2 \alpha t} \sum_{i=1}^{q} \int_{-d_{i}}^{0} e^{2 \alpha u}\left|\eta(u)-\eta^{0}(0)\right|^{2} d u \cdot e^{2 \alpha d_{i}} \\
& +\left.2 q m^{2} l^{2} K^{2} e^{-2 \alpha t} \sum_{i=1}^{q} e^{2 \alpha d_{i}} \int_{0}^{t-d_{i}} e^{2 \alpha u} E\right|^{n} x(u)-\left.\eta^{0}(0)\right|^{2} d u \\
\leqq & \left.2 K^{2} e^{-2 \alpha t}\left[1-\frac{q^{2} m^{2} l^{2}}{2 \alpha}+\frac{q m^{2} l^{2}}{2 \alpha} \sum_{i=1}^{q} e^{2 \alpha d_{i}}\right]\left\|\eta-\eta^{0}\right\|\right|_{C} ^{2} \\
& +\left.2 q m^{2} l^{2} K^{2} e^{-2 \alpha t} \cdot \sum_{i=1}^{q} e^{2 \alpha d_{i}} \cdot \int_{0}^{t} e^{2 \alpha u} E\right|^{\eta} x(u)-\left.\eta^{0}(0)\right|^{2} d u .
\end{aligned}
$$

Set

$$
\begin{gathered}
M=2 K^{2}\left[1-\frac{q^{2} m^{2} l^{2}}{2 \alpha}+\frac{q m^{2} l^{2}}{2 \alpha} \sum_{i=1}^{q} e^{2 \alpha d_{i}}\right], \\
\gamma=-2 q m^{2} l^{2} K^{2} \sum_{i=1}^{q} e^{2 \alpha d_{i}}+2 \alpha .
\end{gathered}
$$

Then, for every $t \geqq r$, we get

$$
\left.e^{2 \alpha t} E\right|^{\eta} x(t)-\left.\eta^{0}(0)\right|^{2} \leqq M\left\|\eta-\eta^{0}\right\|_{c}^{2}+\left.(2 \alpha-\gamma) \int_{0}^{t} e^{2 \alpha u} E\right|^{\eta} x(u)-\left.\eta^{0}(0)\right|^{2} d u
$$

A similar inequality holds for $0 \leqq t \leqq r$; so by Gronwall's lemma it follows that

$$
\left.E\right|^{\eta} x(t)-\left.\eta^{0}(0)\right|^{2} \leqq M\left\|\eta-\eta^{0}\right\|_{c}^{2} e^{-\eta t} \quad \text { for all } t \geqq 0
$$

Note that the inequality (5) means that $\gamma>0$ and so inequality (6) of the theorem is established.

To see that there is at most one absorbing state of (3), observe that if $\tilde{\eta}$ is any (other) 
absorbing state then putting $\eta=\tilde{\eta}$ in (6) immediately gives

$$
\left|\tilde{\eta}(0)-\eta^{0}(0)\right|^{2} \leqq M\left|\tilde{\eta}(0)-\eta^{0}(0)\right|^{2} e^{-\gamma t}
$$

for all $t \geqq 0$. Thus $\tilde{\eta}=\eta^{\circ}$.

The proof of the theorem is complete.

By an easy modification of the above argument, one can prove asymptotic stability in the case of several random delays:

Theorem 4.1. In the stochastic f.d.e. (3), suppose the delays $d_{i}, i=1, \ldots, q$, are $\mathscr{F}_{0}$-measurable P-essentially bounded random variables $d_{i}: \Omega \rightarrow \mathbb{R}^{\geqq 0}$ such that

$$
d_{i}^{0} \equiv \underset{\omega}{\operatorname{essup}} d_{i}(\omega) \leqq r
$$

for all $1 \leqq i \leqq q$. Define $l_{i}, i=1,2, \ldots, q$, by

$$
l_{i}=\inf \left\{\frac{\| g_{i}\left(v_{1}\right)-g_{i}\left(v_{2}\right)||}{\left|v_{1}-v_{2}\right|}: v_{1}, v_{2} \in \mathbb{R}^{n}, \quad v_{1} \neq v_{2}\right\} \text {. }
$$

Suppose that

$$
\sum_{i=1}^{q} l_{i}^{2} e^{2 \alpha d_{i}^{0}}<\frac{\alpha}{q m^{2} K^{2}}
$$

and $\eta^{0}$ is an absorbing state for (3). Then the conclusion (6) of Theorem 1.1 holds with

$$
\begin{gathered}
M=2 K^{2}\left[1+\frac{q m^{2}}{2 \alpha}\left(\sum_{i=1}^{q} l_{i}^{2}\left\{E e^{2 \alpha d_{i}}-1\right\}\right)\right] \\
\gamma=2 \alpha-2 q m^{2} K^{2} \sum_{i=1}^{q} l_{i}^{2} e^{2 \alpha d_{i}^{0}} .
\end{gathered}
$$

Remark. Note that when the $d_{i}$ are all deterministic, condition (26) implies condition (5) of Theorem 1.1.

For distributed random delays, we consider the stochastic f.d.e.

$$
\left\{\begin{aligned}
d x(t) & =H\left(x_{t}\right) d t+\left\{\int_{-r}^{0} g(s, x(t-d(s))) d s\right\} d w(t), \quad t>0 \\
x_{0} & =\eta \in C
\end{aligned}\right.
$$

where $g: J \times \mathbb{R}^{n} \rightarrow L\left(\mathbb{R}^{m}, \mathbb{R}^{n}\right)$ is continuous with $g(s, \cdot)$ globally Lipschitz such that the 
function $l: J \rightarrow \mathbb{R}^{\geqq 0}$,

$$
l(s)=\inf \left\{\frac{\left\|g\left(s, v_{1}\right)-g\left(s, v_{2}\right)\right\|}{\left|v_{1}-v_{2}\right|}: v_{1}, v_{2} \in \mathbb{R}^{n}, v_{1} \neq v_{2}\right\}
$$

belongs to $\mathfrak{L}^{2}(J, \mathbb{R})$. The delay process $d: J \times \Omega \rightarrow \mathbb{R}$ is (Borel $J \otimes \mathscr{F}_{0}$, Borel $\mathbb{R}$ )measurable and essentially bounded such that

$$
d_{s}^{0} \equiv \underset{\omega}{\operatorname{essup}} d(s, \omega) \leqq r
$$

for all $s \in J$. Then (27) has a unique continuous solution through $\eta \in C$ (cf. Mohammed $[13$, pp. 228-230]) given by

$$
x(t)=T_{t}(\eta)(0)+\int_{0}^{t}\left(\widehat{T}_{t-u} \Delta\right)(0) \int_{-r}^{0} g(s, x(u-d(s))) d s d w(u) .
$$

Therefore, we deduce

Theorem 4.2. For the stochastic f.d.e. (27), assume that

$$
\int_{-r}^{0} l(s)^{2} e^{2 \alpha d_{s}^{0}} d s<\frac{\alpha}{r m^{2} K^{2}}
$$

and let $\eta^{0}$ be an absorbing state. Then the conclusion of Theorem 1.1 is satisfied with

$$
M=2 K^{2}+\frac{m^{2} r}{\alpha} \int_{-r}^{0} l(s)^{2}\left\{E e^{2 \alpha d(s)}-1\right\} d s
$$

and

$$
\gamma=2 \alpha-2 m^{2} K^{2} r \int_{-r}^{0} l(s)^{2} e^{2 \alpha d_{s}^{0}} d s
$$

\section{Linear Perturbations}

Consider the linear stochastic f.d.e.

$$
\left\{\begin{aligned}
d x(t) & =H\left(x_{t}\right) d t+G\left(x_{t}\right) d w(t), \quad t>0 \\
x_{0} & =\eta
\end{aligned}\right.
$$

where $H$ is as before (Section 2), $G: C \rightarrow L\left(\mathbb{R}^{m}, \mathbb{R}^{n}\right)$ is continuous linear and $w$ is $m$-dimensional Brownian motion on $\left(\Omega, \mathscr{F},\left(\mathscr{F}_{t}\right)_{t \geq 0}, P\right)$. By the Riesz representation theorem, $G$ corresponds to a Borel, $L\left(\mathbb{R}^{n}, L\left(\mathbb{R}^{m}, \mathbb{R}^{n}\right)\right)$-valued measure $\mu^{\prime}$ on $J$ with total 
variation $v\left(\mu^{\prime}\right)$, viz.

$$
G(\eta)=\int_{-r}^{0} d \mu^{\prime}(s)(\eta(s)), \quad \eta \in C
$$

Note that $\eta^{0}=0$ is clearly an absorbing state for (28). Using the stochastic variation of parameters formula (Theorem 3.1), the solution ${ }^{\eta} x$ of (28) through any $\eta \in C$ is given by

$$
{ }^{\eta} x(t)= \begin{cases}T_{t}(\eta)(0)+\int_{0}^{t}\left(\hat{T}_{t-u} \Delta\right)(0) \int_{-r}^{0} d \mu^{\prime}(s)\left({ }^{\eta} x(u+s)\right) d w(u), & t>0 \\ \eta(t), & -r \leqq t \leqq 0 .\end{cases}
$$

Squaring and taking expectations we get

$$
\begin{aligned}
\left.\left.E\right|^{\eta} x(t)\right|^{2} \leqq & 2 K^{2} e^{-2 \alpha t}\|\eta\|^{2}+2 m^{2} \int_{0}^{t} K^{2} e^{-2 \alpha(t-u)} E\left|\int_{-r}^{0} d \mu^{\prime}(s)\left({ }^{n} x(u+s)\right)\right|^{2} d u \\
\leqq & 2 K^{2} e^{-2 \alpha t}\|\eta\|^{2}+\left.\left.2 m^{2} K^{2} e^{-2 \alpha t} v\left(\mu^{\prime}\right)(J) \int_{-r}^{0} \int_{0}^{t} e^{2 \alpha u} E\right|^{\eta} x(u+s)\right|^{2} d u v\left(\mu^{\prime}\right)(d s) \\
= & 2 K^{2} e^{-2 \alpha t}\|\eta\|^{2}+\left.\left.2 m^{2} K^{2} e^{-2 \alpha t} v\left(\mu^{\prime}\right)(J) \int_{-r}^{0} \int_{s}^{t+s} e^{-2 \alpha s} e^{2 \alpha u} E\right|^{\eta} x(u)\right|^{2} d u v\left(\mu^{\prime}\right)(d s) \\
\leqq & 2 K^{2} e^{-2 \alpha t}\|\eta\|^{2}+\frac{m^{2} K^{2}}{\alpha} e^{-2 \alpha t} v\left(\mu^{\prime}\right)(J)\|\eta\|^{2} \int_{-r}^{0}\left(e^{-2 \alpha s}-1\right) v\left(\mu^{\prime}\right)(d s) \\
& +\left.\left.2 m^{2} K^{2} e^{-2 \alpha t} v\left(\mu^{\prime}\right)(J)\left\{\int_{-r}^{0} e^{-2 \alpha s} v\left(\mu^{\prime}\right)(d s)\right\} \int_{0}^{t} e^{2 \alpha u} E\right|^{\eta} x(u)\right|^{2} d u
\end{aligned}
$$

for all $t \geqq 0, \eta \in C$. Gronwall's lemma then gives

$$
\left.\left.E\right|^{\eta} x(t)\right|^{2} \leqq M\|\eta\|^{2} e^{-\gamma t}, \quad t \geqq 0
$$

where

$$
\begin{gathered}
M=2 K^{2}+\frac{m^{2} K^{2}}{\alpha} v\left(\mu^{\prime}\right)(J) \int_{-r}^{0}\left(e^{-2 \alpha s}-1\right) v\left(\mu^{\prime}\right)(d s) \\
\gamma=2 \alpha-2 m^{2} K^{2} v\left(\mu^{\prime}\right)(J) \int_{-r}^{0} e^{-2 \alpha s} v\left(\mu^{\prime}\right)(d s)
\end{gathered}
$$


In this way we obtain

Theorem 5.1. In the linear stochastic f.d.e. (28), assume that

$$
v\left(\mu^{\prime}\right)(J) \int_{-r}^{0} e^{-2 \alpha s} v\left(\mu^{\prime}\right)(d s)<\frac{\alpha}{m^{2} K^{2}} .
$$

Then the zero solution is globally asymptotically exponentially stable in mean square viz.

$$
\left.\left.E\right|^{\eta} x(t)\right|^{2} \leqq M\|\eta\|^{2} e^{-\gamma t}
$$

for all $t \geqq 0, \eta \in C$, where $M, \gamma>0$ are given by (30) and (31).

Here are some simple examples which are covered by the above theorem:

Let $g: J \times \mathbb{R}^{n} \rightarrow L\left(\mathbb{R}^{m}, \mathbb{R}^{n}\right)$ be continuous with $g(s, \cdot)$ linear for each $s \in J$ and the map

$$
J \ni s \mapsto g(s, \cdot) \in L\left(\mathbb{R}^{n}, L\left(\mathbb{R}^{m}, \mathbb{R}^{n}\right)\right)
$$

is $\mathfrak{L}^{1}$ (Lebesgue-measure). Suppose also that $G: C \rightarrow L\left(\mathbb{R}^{m}, \mathbb{R}^{n}\right)$ is continuous linear. Then for sufficiently small $\varepsilon>0$ the zero solutions of the following stochastic f.d.e.s are globally asymptotically exponentially stable in mean square:

$$
\begin{array}{ll}
d x(t)=H\left(x_{t}\right) d t+\varepsilon G\left(x_{t}\right) d w(t), & t>0 \\
d x(t)=H\left(x_{t}\right) d t+\left\{\int_{-\varepsilon}^{0} g(s, x(t+s)) d s\right\} d w(t), & t>0 \\
d x(t)=H\left(x_{t}\right) d t+\left\{\int_{-r}^{-r+\varepsilon} g(s, x(t+s)) d s\right\} d w(t), & t>0 .
\end{array}
$$

Acknowledgements. The author is grateful to David Elworthy for suggesting some modifications in an earlier version of the manuscript and to Linda Macak for the typing.

\section{REFERENCES}

1. N. Dunford and J. T. Schwartz, Linear Operators, Part I: General Theory (Interscience Publishers, New York, 1967).

2. K. D. Elworthy, Stochastic Differential Equations on Manifolds (LMS Lecture Note Series 70, Cambridge University Press, Cambridge, 1982).

3. A. Friedman, Stochastic Differential Equations and Applications, Vols. 1, 2 (Academic Press, New York-San Francisco-London, 1975).

4. A. Friedman and M. PINSKY, Asymptotic behavior of solutions of linear stochastic differential systems, Trans. Amer. Math. Soc. 181 (1973), 1-22. 
5. J. K. Hale, Theory of Functional Differential Equations (Springer-Verlag, New YorkHeidelberg-Berlin, 1977).

6. J. K. Hale and K. R. Meyer, A Class of Functional Equations of Neutral Type (Mem. Amer. Math. Soc., No. 76, 1967).

7. R. Z. HAS'MINSKII, Stochastic Stability of Differential Equations (Sijthoff and Noordhoff, 1980).

8. K. Itô and M. Nisıo, On stationary solutions of a stochastic differential equation, J. Math. Kyoto University 4 (1964), 1-75.

9. W. Kleimann and L. Arnold, Lyapunov Exponents of Linear Stochastic Systems (Report No. 93, Forschungsschwerpunkt Dynamische Systeme, Universität Bremen, June, 1983).

10. R. Kuвo, The fluctuation-dissipation theorem and Brownian motion, Many-Body Theory (Ed. R. Kubo, Syokabo and Benjamin, 1966), 1-16.

11. H. Kushner, Stochastic Stability and Control (Academic Press, New York, 1967).

12. E. J. McShane, Stochastic Calculus and Stochastic Models (Academic Press, New York-San Francisco-London, 1974).

13. S. E. A. Монаммеd, Stochastic Functional Differential Equations (Research Notes in Mathematics, 99, Pitman Publishing Ltd., Boston-London-Melbourne, 1984).

14. M. PInsky, Stochastic stability and the Dirichlet problem, Communications on Pure and Applied Mathematics 27 (1974), 311-350.

Mathematicś Department

Southern Illinois University at Carbondale

Carbondale, IL 62901 